\title{
Assessing Turbulence Models on the Simulation of Launch Vehicle Base Heating
}

\author{
Pengyu Pu (D) and Yi Jiang (D) \\ School of Aerospace Engineering, Beijing Institute of Technology, Beijing, Beijing 100081, China \\ Correspondence should be addressed to Pengyu Pu; pupengyu7@163.com
}

Received 20 February 2019; Accepted 17 July 2019; Published 22 August 2019

Academic Editor: Antonio Viviani

Copyright (c) 2019 Pengyu Pu and Yi Jiang. This is an open access article distributed under the Creative Commons Attribution License, which permits unrestricted use, distribution, and reproduction in any medium, provided the original work is properly cited.

\begin{abstract}
Launch vehicles suffer from severe base heating during ascents. To predict launch vehicle base heat flux, the computational fluid dynamics (CFD) tools are widely used. The selection of the turbulence model determines the numerical simulation results of launch vehicle base heating, which may instruct the thermal protection design for the launch vehicle base. To assess performances, several Reynolds-averaged turbulence models have been investigated for the base heating simulation based on a four-nozzle launch vehicle model. The finite-rate chemistry model was used for afterburning. The results showed that all the turbulence models have provided nearly identical mean flow properties at the nozzle exit. Menter's baseline (BSL) and shear stress transport (SST) models have estimated the highest collision pressure and have best predicted base heat flux compared to the experiment. The Spalart-Allmaras (SA) model and the renormalization group (RNG) model have performed best in temperature estimation, respectively, in around $r / r_{b}=0 \sim 0.2$ and $r / r_{b}=0.6 \sim 1$. The realizable $k-\varepsilon$ (RKE) model has underestimated the reverse flow and failed to correctly reflect the recirculation in the base region, thus poorly predicted base heating. Among all the investigated turbulence models, the BSL and SST models are more suitable for launch vehicle base heating simulation.
\end{abstract}

\section{Introduction}

Base heating is a severe problem during launch vehicles' ascents [1]. Since 1950s, many launches have failed due to base heating, such as Atlas, Polaris, and Jupiter. This is because severe base heating may damage or destroy mechanical and electrical instruments inside the rocket. For example, in the first flight of Atlas, namely, SM-65A, the hot plume recirculated into the thrust section causing the failure of propellant conducting, then the starvation of LOX shut off the engine. Finally, the rocket fell without adequate thrust. Therefore, it is necessary to carefully design thermal protection for the rocket base from heating.

An efficient thermal protection design relies on an accurate prediction of the heat flux on the walls. However, this is not easy for the complexity of base flow, where recirculation and reversed flow dominate and the hot plume from launch vehicle engines mixes with the ambient cold air, continuously heating the launch vehicle base [2]. Since 1960s, flight test data from Saturn launch vehicles, the space shuttle [3], and data from various wind tunnel tests [4] have been used to predict the convective heating environment in the base region. The total heat flux was classified into convective and radiative base on the physical insight of heating source, and effects of various parameters on base heating, such as nozzle spacing and rocket engine total pressure, were summarized to propose an engineering methodology. However, the methodology depends excessively on flight test data, which is expensive, and the data acquisition is limited. Even if the data is obtained, it is also hard to reveal the flow physics and the specific heat flux distribution on the launch vehicle surface. Although wind tunnel tests [4] could provide more detailed measurements, they still cost much time and resources. Otherwise, a subscale model setup adopted would result in significant discrepancies when analogized to the full-scale configuration. Physical phenomena, such as radiation and flow separation, may not be accurately observed in a subscale wind tunnel test. Therefore, a more economical 
approach providing an insight of the flow field should be implemented for the thermal protection design of the future launch vehicle.

Computational fluid dynamics (CFD), a powerful numerical tool, facilitate launch vehicle base heating predictions, where detailed flow structures and heat flux distributions are easy to obtain by postprocessing. Although CFD facilitates data acquisition, the numerical results almost fully rely on the numerical models involved, which calls for validation. In terms of launch vehicle base heating, turbulence modeling has a crucial effect on the convection dominating base flow, which determines the distribution of heat flux on the bottom surfaces. Due to the tremendous computational consumption, it is infeasible to perform direct numerical simulation (DNS) or large eddy simulation (LES). In practice, Reynolds-averaged turbulence models have been widely used for computational economy. In the past decades, various turbulence models have been adopted in launch vehicle base heating simulation. Mattias [5] adopted the low Reynolds number $k-\varepsilon$ model developed by Launder and Sharma [6] to predict the base flow of a four-nozzle blunt-rocketshaped vehicle. Robert [7] used the Baldwin-Lomax [8] mixing-length model to simulate the base flow induced by exhaust plume impingement. Zhang et al. [9] used the standard two-equation $k-\varepsilon$ model to analyze the base heating of the subscale X-33. The estimated convective heat flux was claimed to agree well with the measurement despite a slight overprediction. Nallasamy et al. [10] utilized the Baldwin-Barth [11] one-equation turbulence model available in the OVERFLOW code to compute the base flow of a four-nozzle clustered rocket engine. Despite qualitative agreement, the numerical heat flux and pressure deviated significantly from Musial's wind tunnel tests' data [4] and the false secondary peak in heat flux was observed. The discrepancies were partly attributed to the inaccuracy of the turbulence model and uncertainties in the base wall temperature. Negishi et al. [12] used the commercial code FLUENT to simulate the base heating environment of the $\mathrm{H}$-II A rocket based on the realizable $k-\varepsilon$ (RKE) turbulence model. Although the simulation well estimated the general heating level, the numerical total heat flux substantially deviated from the flight test, which was thought due to the unimproved mesh refinement and the inaccurate radiation model. However, the defect of the turbulence model adopted was not indicated. Moreover, Mehta et al. [13] adopted Menter's baseline (BSL) turbulence model to investigate numerical sensitivity of base heating predictions based on Musial's tests [4]. Results showed that BSL performed well in base heat flux prediction, while the base pressure was underestimated especially in the low-altitude case. Besides, the BSL model was also used by Patel [14] to predict the base heating environment of Antares.

Although various turbulence models have been individually used to predict launch vehicle base heating, direct comparisons and assessments are lacking between these turbulence models in launch vehicle base heating predictions. This means there might not be enough instructions for turbulence model selection when we estimate launch vehicle base heating. Therefore, an assessment is needed by compar- ing the effect of turbulence models on the launch vehicle base heating simulation. The present work was based on the bellshaped nozzles and low altitude case in wind tunnel tests operated by Musial and Ward [4], and the numerical results for various turbulence models was compared with the test data in order to assess their performances.

\section{Domain and Mesh Setup}

The present study is based on a case of the wind tunnel test in [4]. Figure 1 shows the geometry of the model in this study. A four-nozzle subscale rocket model was used, where $r_{b}=152.4 \mathrm{~mm}, D_{t}=21.463 \mathrm{~mm}, D_{e}=74.676 \mathrm{~mm}$, $L / D_{e}=1.53$, and $D_{s} / D_{e}=1.67$. Figure 2 shows the mesh generated for computation. Based on the geometric symmetry, a $1 / 8$ model was adopted to reduce computational cost. Unstructured cell meshes were used to refine the region inside the nozzle and near the bottom and prism cell meshes to resolve the boundary layer. The thickness of the first boundary layer mesh is $4 \mu \mathrm{m}$ to guarantee $y^{+}<1$.

To ensure grid independence, three sets of meshes ( 2 million, 3.5 million, and 5 million) were calculated with a simple equivalent gas model without reactions. The radial base heat flux (along the radial line shown in Figure 1) is compared in Figure 3. It has been indicated that the derivation was less than $5 \%$ between 3.5 million and 5 million cell meshes, so the grid with 3.5 million cells was applied for economy, which is accurate enough to compare performances of turbulence models.

\section{Computational Model Setup}

3.1. Computing Method. An implicit density-based solver, which is appropriate for highly compressible flow, was utilized with a time marching method to approach the steady state. The Reo flux difference splitting scheme [15] was adopted for discontinuity of the shocks due to the supersonic upstream air and rocket engines' exhaust plume. All the convection terms in transport equations were discretized by a second-order upwind scheme with adequate fidelity for engineering, and the gradient was evaluated by the least squares of the corresponding variable at the neighbor cell center. Besides, convergence acceleration was applied for the stretched boundary layer meshes.

3.2. Gas Model. Two aspects determine gas modeling. On the one hand, the specific heat of each component in the gas mixture varies with temperature, which is coupled with heat transfer in the fluid and through the wall. Therefore, a polynomial fit curve was adopted to account for the variation. On the other hand, afterburning occurs when $\mathrm{CO}$ and $\mathrm{H}_{2}$ contact the ambient fresh air, increasing the local temperature. Thus, finite-rate chemistry was incorporated. To simplify chemical reaction computations, the JP- 4 was approximated by RP-1 to utilize the widely accepted wet-CO [16], which significantly reduced the number of reacting components involved while providing adequate accuracy. Table 1 shows the elementary reaction system employed in the present study, where the parameters for the Arrhenius equation were 

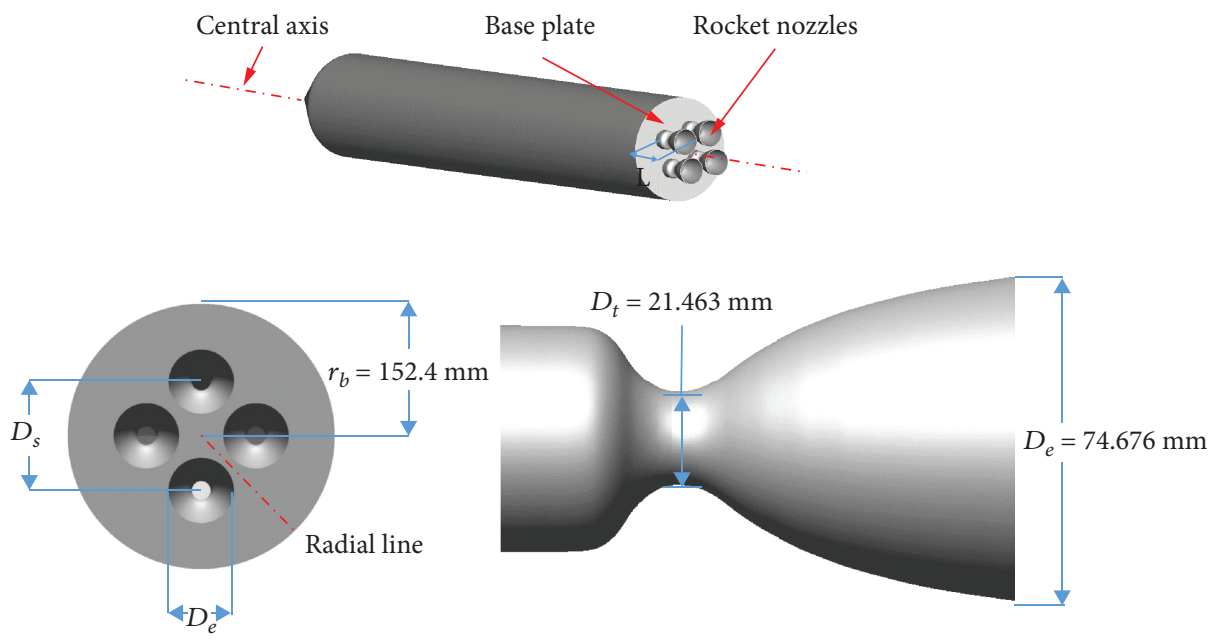

FIgURE 1: Geometry of the four-nozzle rocket.
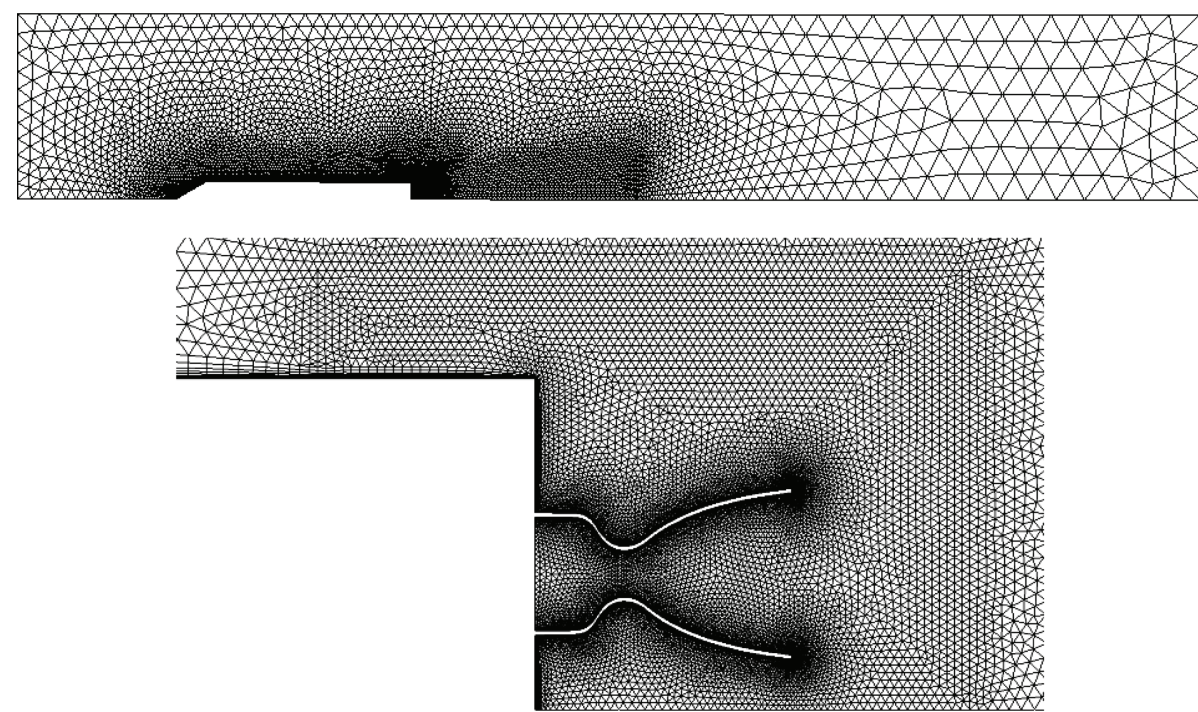

FIgURE 2: Mesh generated for computation.

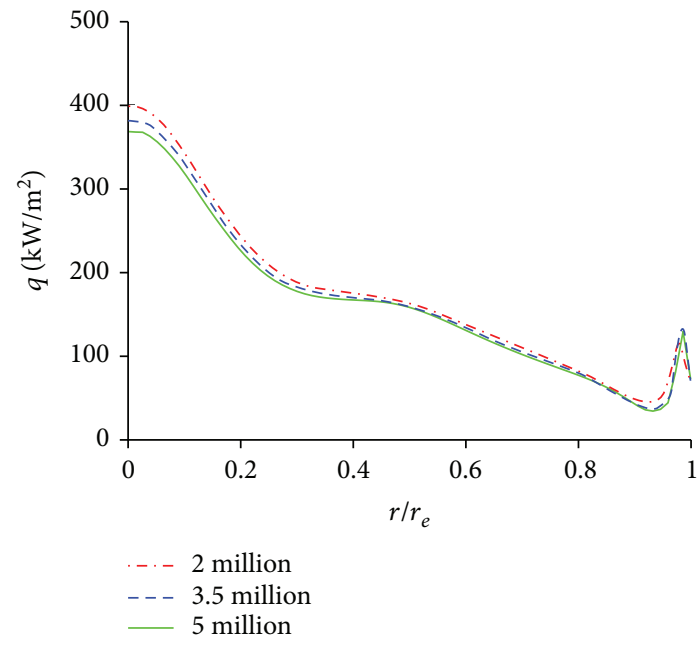

FIGURE 3: Grid convergence verification. obtained from NIST Chemical Kinetics Database. The initial mass fraction of each component was calculated by a thermodynamic equilibrium code based on the minimum Gibbs free energy. Finally, a multispecies mixture model was used to compute finite-rate chemistry consisting of 9 components from the combustion of the rocket engine propellant and the atmosphere, including $\mathrm{CO}_{2}, \mathrm{CO}, \mathrm{H}_{2} \mathrm{O}, \mathrm{H}_{2}, \mathrm{OH}, \mathrm{O}_{2}, \mathrm{~N}_{2}$, $\mathrm{H}$, and $\mathrm{O}$.

3.3. Turbulence Modeling. The RANS turbulence model selected for numerical simulations may affect the spread of the hot gas [17], or rather, the predicted heat flux distribution on the rocket base partly depends on the selection of the turbulence model. Given, respectively, the round jet anomaly and the freestream sensitivity, neither the standard $k-\varepsilon$ model nor the standard $k$ - $\omega$ model has been adopted to estimate launch vehicle base heating. The RANS models investigated in this study are the Spalart-Allmaras (SA), realizable $k-\varepsilon$ 
TABLE 1: RP-1/LOX plume finite-rate reactions.

\begin{tabular}{lccc}
\hline Reactions & $A(\mathrm{~m}, \mathrm{kmol}, \mathrm{s})$ & $b$ & Ea $(\mathrm{J} / \mathrm{kmol})$ \\
\hline $\mathrm{H}+\mathrm{O}_{2} \longleftrightarrow \mathrm{OH}+\mathrm{O}$ & $2.07 \times 10^{11}$ & -0.097 & $6.29 \times 10^{7}$ \\
$\mathrm{O}+\mathrm{H}_{2} \longleftrightarrow \mathrm{H}+\mathrm{OH}$ & 38.7 & 2.7 & $2.621 \times 10^{7}$ \\
$\mathrm{OH}+\mathrm{H}_{2} \longleftrightarrow \mathrm{H}+\mathrm{H}_{2} \mathrm{O}$ & $2.17 \times 10^{5}$ & 1.52 & $1.45 \times 10^{7}$ \\
$\mathrm{OH}+\mathrm{OH} \longleftrightarrow \mathrm{O}+\mathrm{H}_{2} \mathrm{O}$ & 33.5 & 2.42 & $-8.06 \times 10^{6}$ \\
$\mathrm{H}+\mathrm{H}+\mathrm{M} \longleftrightarrow \mathrm{H}_{2}+\mathrm{M}$ & $1.01 \times 10^{11}$ & -0.6 & 0 \\
$\mathrm{H}+\mathrm{O}+\mathrm{M} \longleftrightarrow \mathrm{OH}+\mathrm{M}$ & $5 \times 10^{12}$ & -1 & 0 \\
$\mathrm{O}+\mathrm{O}+\mathrm{M} \longleftrightarrow \mathrm{O}_{2}+\mathrm{M}$ & $5.4 \times 10^{7}$ & 0 & $-7.4 \times 10^{6}$ \\
$\mathrm{H}+\mathrm{OH}+\mathrm{M} \longleftrightarrow \mathrm{H}_{2} \mathrm{O}+\mathrm{M}$ & $5.56 \times 10^{16}$ & -2 & 0 \\
$\mathrm{CO}+\mathrm{OH} \longleftrightarrow \mathrm{CO}_{2}+\mathrm{H}$ & $4.76 \times 10^{4}$ & 1.23 & $2.9309 \times 10^{5}$ \\
$\mathrm{CO}+\mathrm{O}_{2} \longleftrightarrow \mathrm{CO}_{2}+\mathrm{O}$ & $2.5 \times 10^{9}$ & 0 & $2 \times 10^{8}$ \\
$\mathrm{CO}+\mathrm{O}+\mathrm{M} \longleftrightarrow \mathrm{CO}_{2}+\mathrm{M}$ & $1.54 \times 10^{9}$ & 0 & $1.25 \times 10^{7}$ \\
$\mathrm{O}_{2}+\mathrm{H}_{2} \longleftrightarrow \mathrm{OH}_{+} \mathrm{OH}$ & $2.5 \times 10^{9}$ & 0 & $1.87 \times 10^{5}$ \\
\hline
\end{tabular}

(RKE), RNG $k-\varepsilon$, Menter's BSL $k-\omega$, and SST $k-\omega$ models. The turbulent Prandtl number $\operatorname{Pr}_{t}$ is set as 0.85 for all the turbulence models involved.

3.3.1. Spalart-Allmaras (SA) Model. The Spalart-Allmaras model [18], which is widely used in analysis of aerodynamics force, is a one-equation turbulence model solving the singletransport equation of turbulent kinetic viscosity $\tilde{v}$. The SA model performs well in wall-bounded flows and boundary layers subjected to adverse pressure gradient. This model was involved to investigate its feasibility for launch vehicle base heating prediction.

Eq. (1) shows the transport equation for the SpalartAllmaras model.

$$
\begin{aligned}
& \frac{\partial}{\partial t}(\rho \tilde{v})+\frac{\partial}{\partial x_{i}}\left(\rho \tilde{v} u_{i}\right) \\
& \quad=G_{v}+\frac{1}{\sigma_{v}}\left[\frac{\partial}{\partial x_{j}}\left\{(\mu+\rho \tilde{v}) \frac{\partial \tilde{v}}{\partial x_{j}}\right\}+C_{b 2} \rho\left(\frac{\partial \tilde{v}}{\partial x_{j}}\right)^{2}\right]-Y_{v}
\end{aligned}
$$

where $G_{v}$ is the production of turbulent viscosity and $Y_{v}$ is the destruction of turbulent viscosity in the near-wall region due to wall blocking and viscous damping. $\sigma_{\tilde{v}}$ and $C_{b 2}$ are the model constants, and $v$ is the molecular kinetic viscosity.

The $y^{+}$-insensitive Menter-Lechner wall treatment was utilized, blending all solution variables from viscous sublayer to the logarithmic layer based on the value of $y^{+}$.

3.3.2. Realizable $k-\varepsilon$ Model. The realizable $k-\varepsilon$ (RKE) model [19] is a modification of the standard two-equation $k-\varepsilon$ model, which describes turbulence by turbulence kinetic energy $k$ and turbulent dissipation rate $\varepsilon$, providing an alternative formulation for the turbulent viscosity and considering the transport of the mean-square vorticity fluctuation in the $\varepsilon$ transport equation. This model has shown improvement in the flow where exists large streamline curvature and rotation.

Eqs. (2) and (3) show the transport equations of the realizable $k-\varepsilon$ model.

$$
\begin{aligned}
\frac{\partial}{\partial t}(\rho k)+\frac{\partial}{\partial x_{i}}\left(\rho k u_{i}\right)= & \frac{\partial}{\partial x_{j}}\left[\left(\mu+\frac{\mu_{t}}{\sigma_{k}}\right) \frac{\partial k}{\partial x_{j}}\right]+G_{k}-\rho \varepsilon, \\
\frac{\partial}{\partial t}(\rho \varepsilon)+\frac{\partial}{\partial x_{i}}\left(\rho \varepsilon u_{i}\right)= & \frac{\partial}{\partial x_{j}}\left[\left(\mu+\frac{\mu_{t}}{\sigma_{\varepsilon}}\right) \frac{\partial \varepsilon}{\partial x_{j}}\right] \\
& +\rho C_{1} S \varepsilon-\frac{\rho C_{2} \varepsilon^{2}}{k+\sqrt{\nu \varepsilon}}, \\
\mu_{t}= & \frac{\rho C_{\mu} k^{2}}{\varepsilon},
\end{aligned}
$$

where $\mu_{t}$ is the turbulent dynamic viscosity, expressed as Eq. (4), $\sigma_{k}$ and $\sigma_{\varepsilon}$ are turbulent Prandtl numbers, respectively, for $k$ and $\varepsilon, G_{k}$ is the generation of $k$ due to the mean velocity gradients, $C_{2}$ is a constant, and $C_{1}=\max$ [ 0.43, $(\eta /(\eta+5))], \quad \eta=S k / \varepsilon . S=\sqrt{2 S_{i j} S_{i j}} . C_{\mu}$ is related to the magnitude of mean rate-of-rotation tensor $\Omega$ and mean rate-of-strain tensor $S$.

3.3.3. RNG $k-\varepsilon$ Model. The RNG $k-\varepsilon$ model [20] originates from the well-known renormalization group (RNG) theory, which provides an analytical formula for turbulent Prandtl numbers. The RNG model introduces the conception of effective viscosity and the inverse effective Prandtl numbers. The formula of the effective viscosity varies with the eddy scale, and the effective Prandtl numbers are related to the molecular Prandtl numbers.

3.3.4. BSL $k$ - $\omega$ Model. The BSL $k$ - $\omega$ model is a modification of the Wilcox $k-\omega$ model. This model was developed by Menter [21] to blend the $k-\omega$ model in the near-wall region with the $k-\varepsilon$ model which is insensitive to the freestream conditions in the far field.

Eq. (5) shows the transport equations for the BSL model:

$$
\begin{aligned}
\frac{\partial}{\partial t}(\rho k)+\frac{\partial}{\partial x_{i}}\left(\rho k u_{i}\right)= & \frac{\partial}{\partial x_{j}}\left[\left(\mu+\frac{\mu_{t}}{\sigma_{k}}\right) \frac{\partial k}{\partial x_{j}}\right]+G_{k}-Y_{k}, \\
\frac{\partial}{\partial t}(\rho \omega)+\frac{\partial}{\partial x_{i}}\left(\rho \omega u_{i}\right)= & \frac{\partial}{\partial x_{j}}\left[\left(\mu+\frac{\mu_{t}}{\sigma_{\omega}}\right) \frac{\partial \omega}{\partial x_{j}}\right] \\
& +G_{\omega}-Y_{\omega}+D_{\omega},
\end{aligned}
$$

where $G_{k}$ and $G_{\omega}$ are the production, respectively, of $k$ and $\omega$. $Y_{k}$ and $Y_{\omega}$ represent the dissipation of $k$ and $\omega$ due to turbulence, and $D_{\omega}$ is the cross-diffusion term for $\omega . \sigma_{k}$ and $\sigma_{\omega}$ 
represent the turbulent Prandtl numbers of $k$ and $\omega$, which are formulated as

$$
\begin{gathered}
\sigma_{k}=\frac{1}{\left(F_{1} / \sigma_{k, 1}\right)+\left(\left(1-F_{1}\right) / \sigma_{k, 2}\right)}, \\
\sigma_{\omega}=\frac{1}{\left(F_{1} / \sigma_{\omega, 1}\right)+\left(\left(1-F_{1}\right) / \sigma_{\omega, 2}\right)} .
\end{gathered}
$$

The blending function $F_{1}$ is given by

$$
\begin{aligned}
& F_{1}=\tanh \left(\Phi_{1}^{4}\right) \\
& \Phi_{1}=\min \left[\max \left(\frac{\sqrt{k}}{0.09 \omega y}, \frac{500 \mu}{\rho y^{2} \omega}\right), \frac{4 \rho k}{\sigma_{\omega, 2} D_{\omega}^{+} y^{2}}\right]
\end{aligned}
$$

where $y$ is the distance to the next surface and $D_{\omega}^{+}$is the positive portion of $D_{\omega}$.

3.3.5. SST $k$ - $\omega$ Model. The SST model is another modification proposed by Menter et al. [21, 22], which includes all the refinements of the BSL model. The SST model was theoretically more appropriate for adverse pressure gradient flows. The differences lie in the formulation of $\mu_{t}$ and the blending function $F_{2}$, which are

$$
\begin{aligned}
\mu_{t} & =\frac{\rho k / \omega}{\max \left[1 / \alpha^{*}, S F_{2} / a_{1} \omega\right]}, \\
F_{2} & =\tanh \left(\Phi_{2}^{2}\right), \\
\Phi_{2} & =\max \left[2 \frac{\sqrt{k}}{0.09 \omega y}, \frac{500 \mu}{\rho y^{2} \omega}\right],
\end{aligned}
$$

where $\alpha^{*}$ and $a_{1}$ are constants.

3.4. Boundary Conditions. The present simulations were performed on the conditions of the lower-altitude case in [4], as shown in Table 2. The mass fractions of plume components shown in Table 3 were calculated as the inlet conditions of the exhaust plume based on thermodynamic equilibrium.

\section{Results and Discussion}

Due to the lack of measurements, the numerical results are to be validated by the experimental data provided by Musial's wind tunnel test [4], only composed of data on base heat flux, pressure, and near-base gas temperature at several monitor points. Although not subtle enough for accurate predictions academically, it is convincing adequately to compare the performances of the turbulence models.

4.1. Nozzle Exit. According to previous investigations [13], the launch vehicle base heating could be significantly affected by the nozzle internal boundary layer flows, which would be manifested by the parameter distributions at the nozzle exits. Figure 4 shows the parameters along the radius of nozzle exits from the center to the edge for different turbulence models. It has been indicated that there are no significant
TABLE 2: Boundary conditions.

\begin{tabular}{lc}
\hline Parameters & Values \\
\hline$P_{c}$ & $17.5 \mathrm{MPa}$ \\
$T_{c}$ & $3825.1 \mathrm{~K}$ \\
$P_{\infty}$ & $12209.5 \mathrm{~Pa}$ \\
$T_{\infty}$ & $297.7 \mathrm{~K}$ \\
$M a_{\infty}$ & 2.75 \\
\hline
\end{tabular}

TABLE 3: Mass fractions of plume components.

\begin{tabular}{lc}
\hline Formulae & Mass fractions \\
\hline $\mathrm{CO}_{2}$ & 0.2033 \\
$\mathrm{CO}$ & 0.4946 \\
$\mathrm{H}_{2} \mathrm{O}$ & 0.2533 \\
$\mathrm{H}_{2}$ & 0.0135 \\
$\mathrm{O}_{2}$ & 0.0052 \\
$\mathrm{OH}$ & 0.0253 \\
$\mathrm{H}$ & 0.0015 \\
$\mathrm{O}$ & 0.0031 \\
\hline
\end{tabular}

discrepancies in all the parameters except turbulence viscosity, which has been expected as the turbulence intensity should be as low as about $1 \% \sim 2 \%$ at the nozzle exit [23] and the turbulence have moderate effect here. Moreover, finite-rate chemistry changed the mass fractions of plume components. It has been shown that the mass fraction of $\mathrm{CO}_{2}$ at the nozzle exit was significantly higher than that in the combustion chamber. This has indicated the formation of $\mathrm{CO}_{2}$ along the nozzle internal flow for the relative low temperature at the divergent sections which promoted the exothermic reactions. A large amount of heat was released through these reactions, thus implied the potentially higher reverse flow temperature.

4.2. Base Flow. While the base heating originates from the high temperature of the exhaust plume, the base heat flux distribution also depends on the base flow structures, which vary among rocket nozzle configurations and will be influenced by ambient conditions [3]. In terms of numerical computation, the simulated flow structures may be significantly affected by the turbulence model in use. Therefore, it is necessary to compare the flow structures derived from turbulence models in the base region.

According to the theory on the four-nozzle rocket base flow phenomenon $[4,13]$, the plumes collided together after flowing out of the nozzles, which resulted in a high-pressure region and reversed the downstream plume. The reverse flow then impinged on the rocket base plate before flowing along the base wall to form the wall jet. The wall jet then mixed with the upstream air, which generated the recirculation vortices in the base region. The recirculation continuously entrained the cold air into the base region to cool down the base plate. Figure 5 compares the base flow structures derived from the 


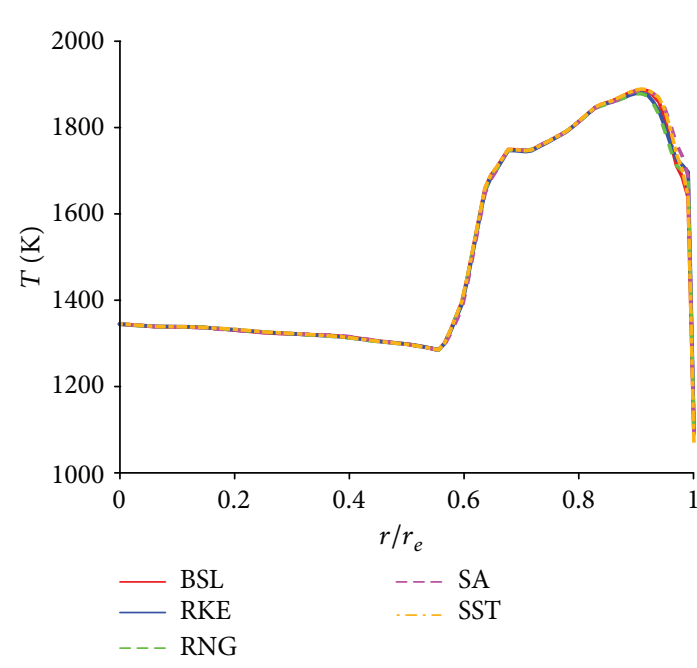

(a) Temperature

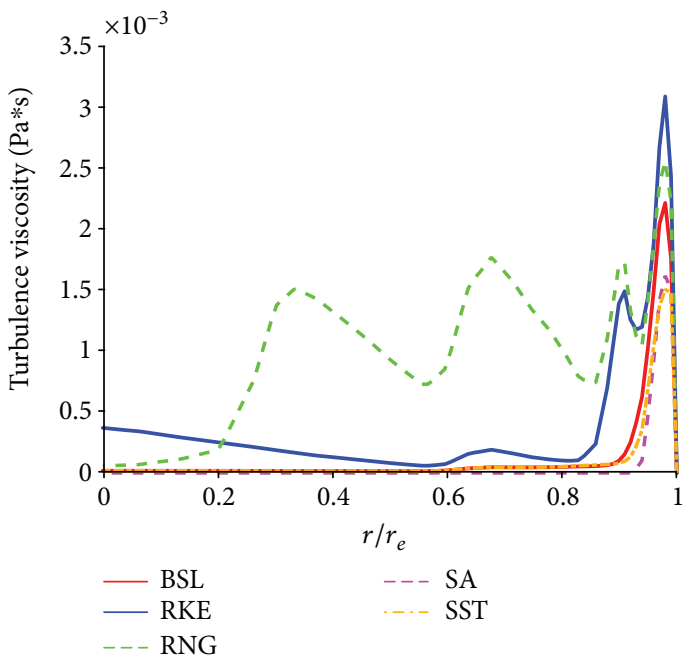

(c) Turbulence viscosity

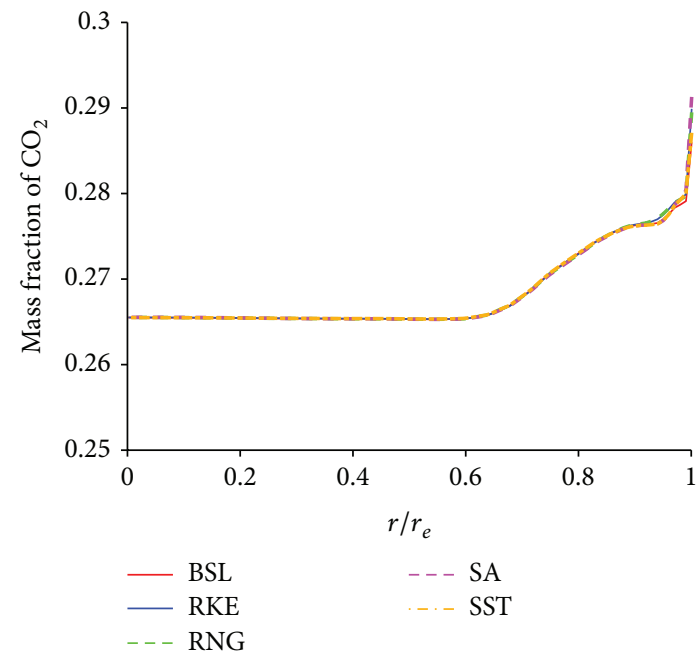

(e) Mass fraction of $\mathrm{CO}_{2}$

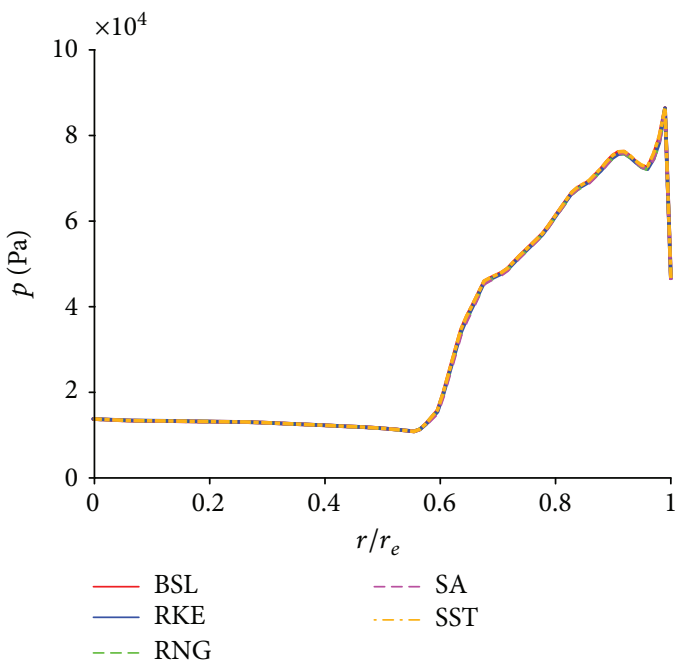

(b) Pressure

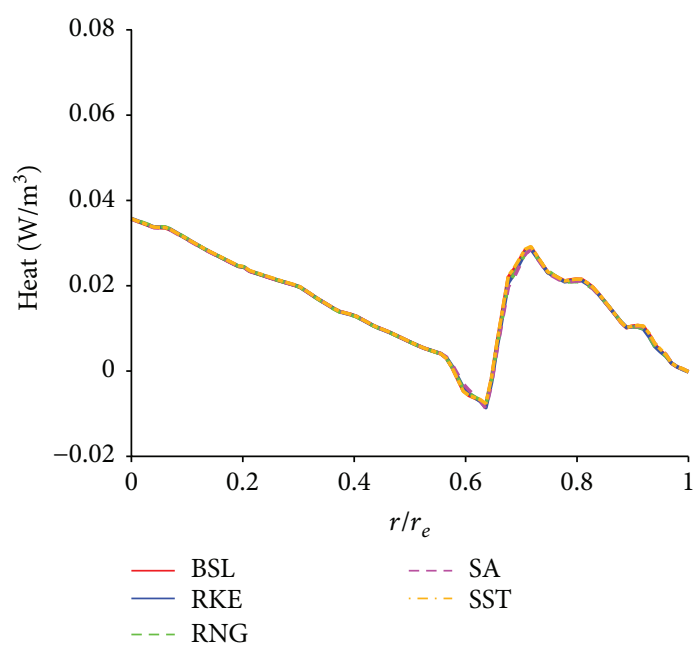

(d) Reaction heat

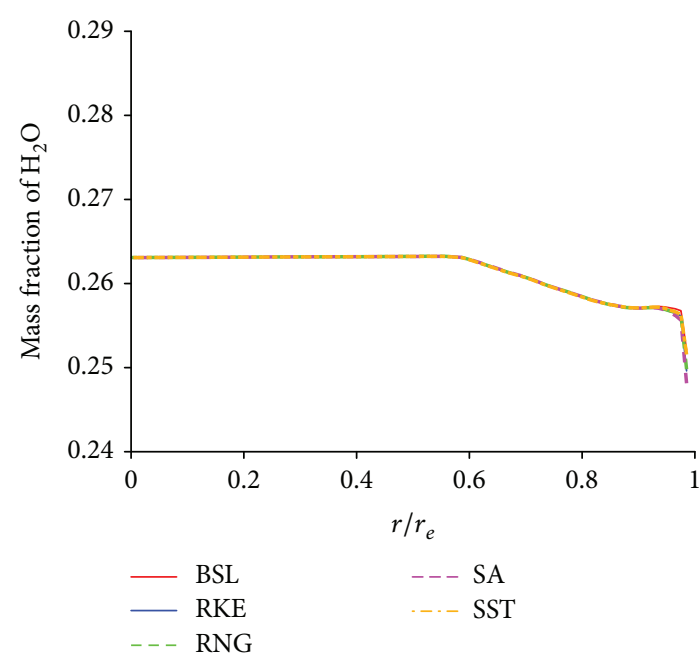

(f) Mass fraction of $\mathrm{H}_{2} \mathrm{O}$

FIgURE 4: Radial parameter distribution at the nozzle exit for different turbulence models. 


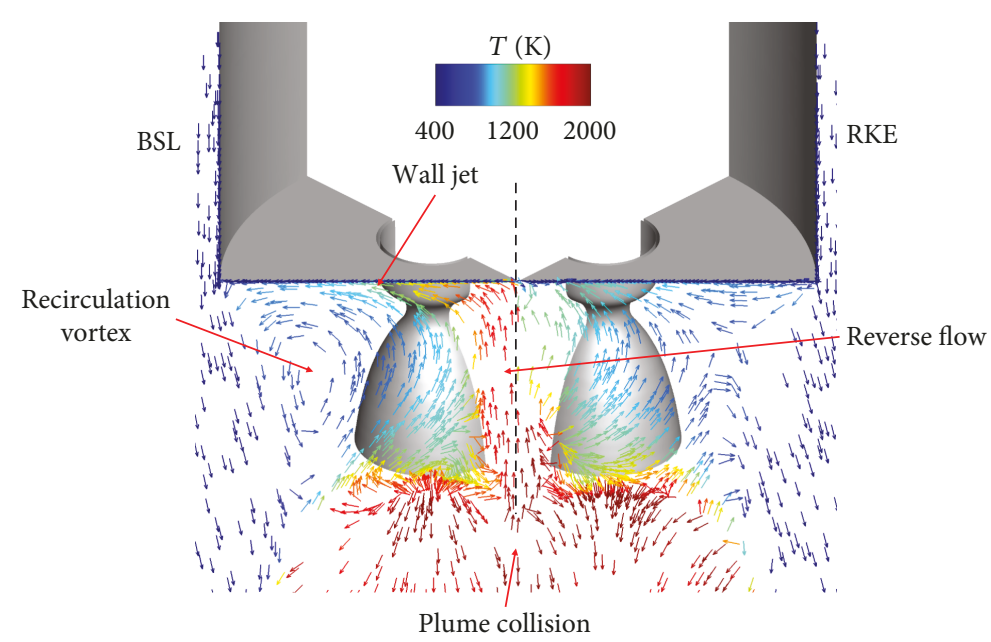

FIgUre 5: Base flow structures for BSL (left half) and RKE (right half).

BSL and RKE turbulence models. Flow structures for other turbulence models have not been exhibited for their similarity to BSL. It has been shown that the BSL model has well predicted the reverse flow, the wall jet and the recirculation vortex, while the RKE model has failed to predict the wall jet direction or the recirculation correctly. Furthermore, the temperature of reverse flow was underestimated by RKE compared to that by BSL. This implies that the RKE model may not apply in launch vehicle base heating predictions. Further comparisons on characteristics of base flow are discussed below.

4.2.1. Plume Collision and Reverse Flow. One of characteristics of the reverse flow is its reverse velocity that affects the intensity of impingement on the base. It has been indicated that the reverse flow results from the large pressure gradient due to the collision among plumes $[4,13]$. That is, the velocity of reverse flow depends on the collision point and the collision pressure. The collision point is defined as the point with the maximum pressure due to collision, and collision pressure is defined as the local pressure of the collision point. Figure 6(a) shows the pressure along the central axis of the rocket from the bottom. Results show that Menter's BSL and SST models have provided the highest collision pressure, approximately 5.6 times the far-field condition, higher than that of RKE by nearly $70 \%$ of the ambient pressure. Furthermore, these two turbulence models have predicted the collision point closest to the rocket base, which means the largest plume expansion angle. Besides, RNG and SA have also estimated higher collision pressure than RKE, while the predicted collision points were farther from the base, nearly $10 \%$ of the base radius than that of the BSL and SST models. Figure 6(b) compares the axial velocity component $U_{z}$ provided by different turbulence models, where the positive value refers to the direction of reverse flow. The reverse point is defined where the sign of $U_{z}$ changes, and the reverse velocity is defined as the peak of $U_{z}$ in reverse flow. In general, higher collision pressure leads to smaller distance from the reverse point to the collision point, due to the more significant acceleration. It has been manifested that the RKE model underpredicted the reverse velocity by more than $40 \%$ than others and the estimated reverse point was closest to the base plate. The underprediction results from the lower estimated collision pressure, and the more adjacent reverse point is also due to the relatively closer collision point. From this point of view, the RNG model has predicted the farthest collision point and the estimated collision pressure was high enough, thus deriving the farmost reverse point, as shown in Figure 6(b). Also, although the SA model has underpredicted the collision pressure than BSL and SST, the estimated collision point was farther from the bottom; hence, no discrepancies exist in reverse point position. Moreover, Figure 6(b) has also indicated the excessive dissipation of the reverse flow velocity in the RKE model as the reverse flow approached the base plate, where the stagnation occurred nearly half way of the distance from the reverse point to the rocket bottom. The reverse flow velocity of the SA model was lower than that of BSL, RNG, and SST. As the heat transfer to the base plate is regarded as convection dominated, the declines of reverse flow velocity implied the decrease in base heat flux.

Figure 7 (a) shows the turbulence viscosity along the central axis. Generally, all the turbulence models have resulted a maximum of turbulence viscosity around the collision point, where the RKE model has significantly overestimated the turbulence viscosity peak, which is the main derivation of the excessive dissipation in reverse flow velocity. Figure 7(b) shows the magnitude of the strain rate tensor S. Obviously, the peak of $S$ approximately corresponds to the maximum of turbulence viscosity. It may be inferred that the overprediction on turbulence viscosity by RKE could result from the $S$ involved in the formulation of $C_{\mu}$ adopted in Eq. (4), where the "realizable" correction may have distorted the turbulence viscosity.

Another significant characteristic of reverse flow that determines the base heat flux distribution is the reverse flow temperature, which is coupled with afterburning resulting from the mixture between high-temperature plume and oxygen-rich air. Figure 8 shows the mean static temperature 


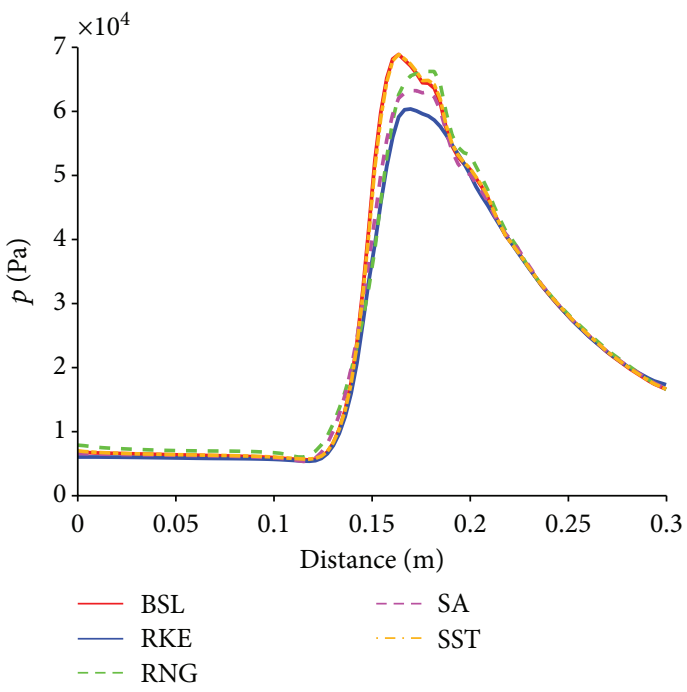

(a) Pressure

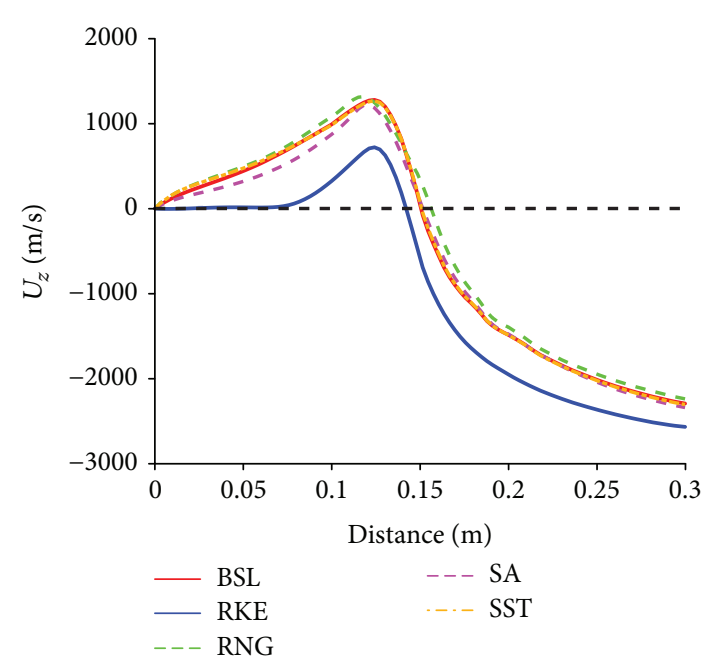

(b) Axial velocity component

FIGURE 6: Pressure and axial velocity component distribution along the rocket center axis from the bottom.

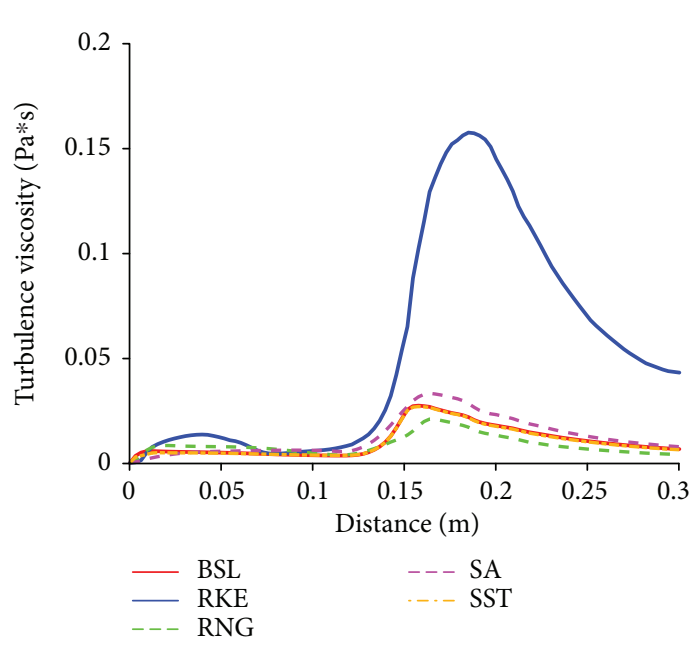

(a) Turbulence viscosity

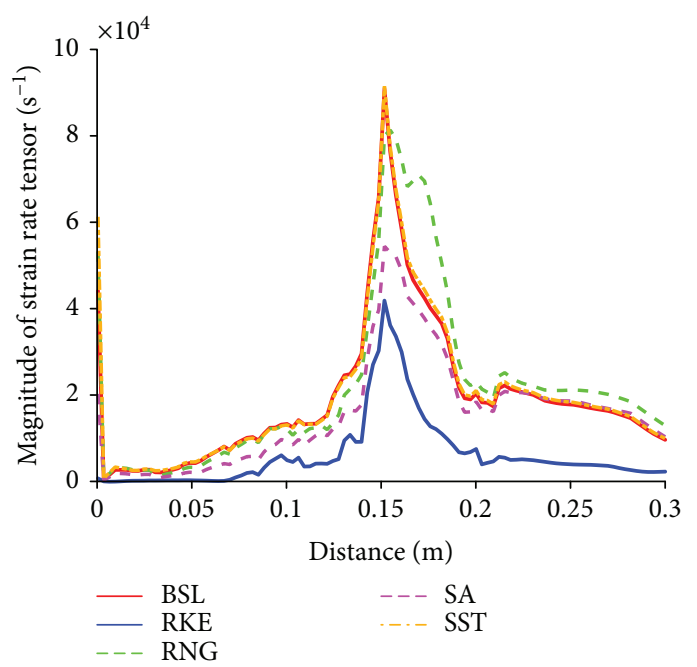

(b) Magnitude of the strain rate tensor

FIGURE 7: Turbulence viscosity and magnitude of the strain rate tensor along the central axis of the rocket.

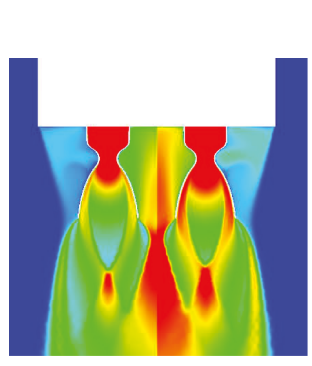

BSL

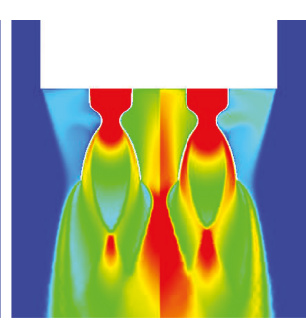

RKE

(b)

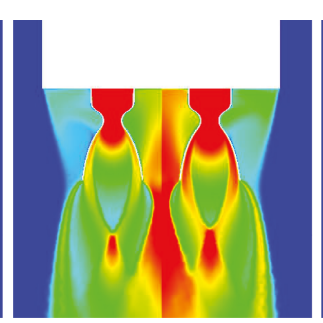

RNG

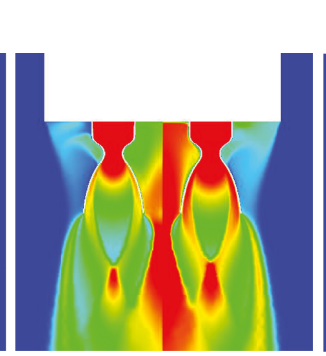

SA

(d)

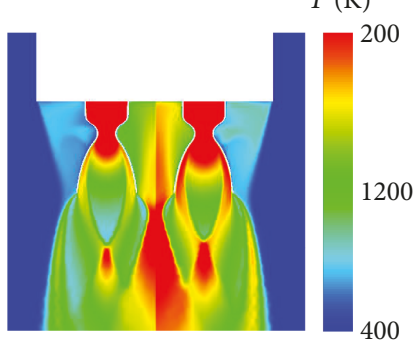

SST

(e)

FiguRE 8: Static temperature contours between frozen flow (left half) and finite-rate chemistry (right half) for different turbulence models. 


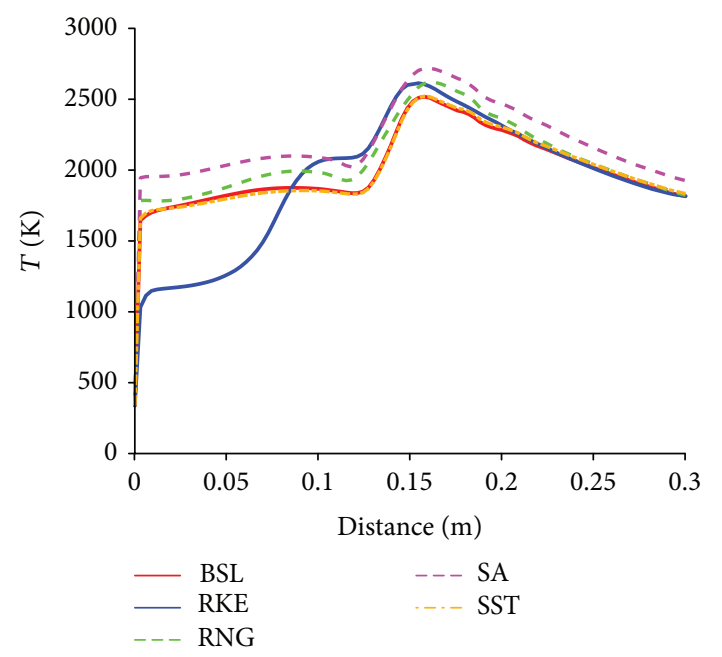

Figure 9: Static temperature along the central axis of the rocket.

field for the investigated turbulence models, and the results of frozen flow are included for comparison. Generally, finiterate chemistry has significantly increased the temperature in the nozzle's divergent sections and near the rocket center axis. Among all the turbulence models involved, the SA model provided the highest temperature of the reverse flow and the RNG model estimated higher temperature in the recirculation region than the others. Furthermore, it has been indicated that the reverse flow temperature of the RKE model was somewhat "cutoff," which links to the misestimated flow structures shown in Figure 5. Figure 9 shows the axial static temperature from the rocket base. It has been suggested that the maximum of temperature was located near the collision point and the reverse flow temperature for RKE dropped steeply when approaching to the base, decreasing by approximately $30 \%$ of that for BSL and SST, despite the higher temperature at the collision point. Besides, higher reverse flow temperature was also predicted by RNG without a substantial near-wall drop. Figure 10 shows the distribution of $\mathrm{CO}_{2}$ in the base region, which indicates the spread of high-temperature exhaust. It has been shown that all the turbulence models except RKE have provided a high level of $\mathrm{CO}_{2}$ mass fraction in the reverse flow. This is true because the reverse flow originates from the plume collision. In other words, the RKE model has misestimated plume transport thus underpredicting reverse flow temperature shown in Figure 8(b). Furthermore, the RNG model has provided the highest $\mathrm{CO}_{2}$ mass fraction in the recirculation region, corresponding to the relatively higher local temperature in Figure 8(c) with more exhaust involved.

4.2.2. Impingement and Wall Jet. The upstream reverse flow impinged on the rocket base plate before the impinged gas stagnated near the base center, forming a high-pressure region. The large pressure gradient then drove the hot gas to spread outward along the base wall to generate the so-called wall jet. According to previous investigations, jet impingement could significantly promote heat transfer for its highly convective nature [24]. The convective characteristics, such as flow velocity and turbulence level of the wall jet, determined the heat transfer from gas to base. Figure 11 shows the magnitude of velocity and turbulent thermal conductivity $\kappa_{t}$ along the radius $12.7 \mathrm{~mm}$ off the base, which is located inside the wall jet identical to the temperature sensors in [4]. It has been indicated that the RKE model has underestimated the velocity near the central axis, identical to the low reverse flow velocity mentioned before. Apart from RKE, all the turbulence models have predicted the attenuation in velocity from the center as well as the subsequent increase to a relatively constant level from $r / r_{b}=0.4$. Generally, the RNG model has provided the fastest wall jet flow, although slightly slower than SA in $r / r_{b}=0.4 \sim 0.8$. Besides, despite the favorable agreement between BSL and SST in the discussion above, the velocity in the central area provided by SST was higher than that by BSL by about $30 \%$, which indicates potentially more significant convective heat transfer of the SST model. Furthermore, the RKE model has estimated $\kappa_{t}$ not too bad, within the same level as the others, although the second peak was prepositioned. All the turbulence models have predicted the central peak of $\kappa_{t}$, where the SA model provided the lowest $\kappa_{t}$, approximately half of that by SST. The RKE and RNG model estimated the highest central peak, while the predicted $\kappa_{t}$ in $r / r_{b}>0.5$ was at least $1 / 3$ lower than that of the other three models, as shown in Figure 11(b).

4.3. Parameter Distributions on the Base Plate. The ultimate goal of launch vehicle base heating computation is to predict the base heat flux distribution, which is the criterion to assess the performances of various turbulence models. Parameters on the base plate are shown below, and the relationships between their distributions and the base flow mentioned above are discussed.

4.3.1. Base Heat Flux. Figure 12 shows the heat flux distribution on the base plate for different turbulence models. All the models except RKE have suggested the pronounced local extremum at the base center, while the SA model has relocated the global maximum heat flux to around $x=0.1$ $\mathrm{m}, y=0 \mathrm{~m}$. Generally, base heat flux almost monotonously decreased from the center to the edge. Comparing to BSL and SST, which have indicated great similarity, the RNG model has predicted the higher heat transfer rate at outer regions. This overprediction was correlated to the higher local gas temperature due to the overinvolved exhaust, as discussed above. Furthermore, the RKE model has failed to estimate the central peak as expected and has underestimated the base heating due to the lower predicted reverse flow temperature as well as the weaker reverse flow intensity.

Figure 13 compares the numerical radial base heat flux with the experimental data in Musial's wind tunnel tests [4]. For frozen flow, the RKE model has failed to predict qualitatively the central peak heat flux and prominently underpredicted quantitatively the overall base heating, at best $50 \%$ lower than the experimental data at around $r / r_{b}=0.4$. The SA model either has failed to manifest the qualitative distribution of base heat flux, despite a better performance than RKE. Moreover, the other three turbulence models 


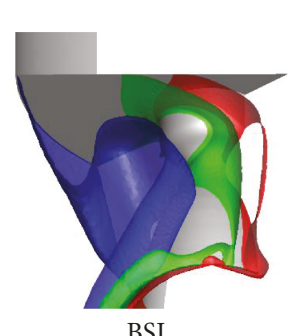

(a)

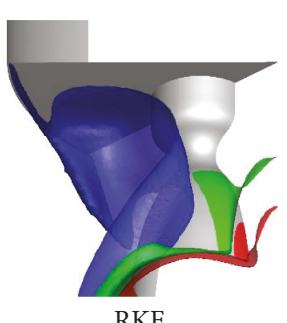

(b)

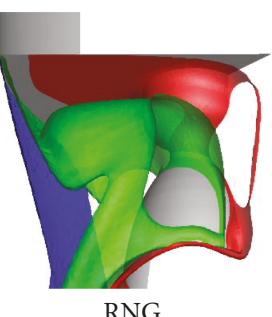

(c)

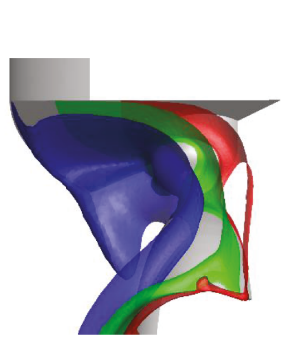

SA

(d)

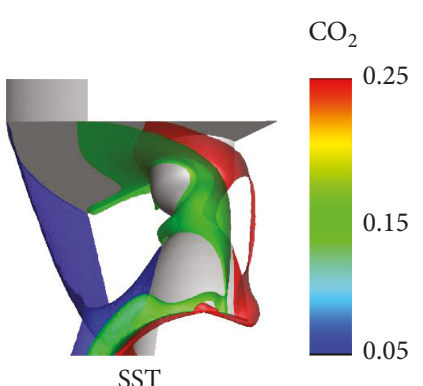

(e)

FIGURE 10: Isosurfaces of $\mathrm{CO}_{2}$ mass fractions in the base region.

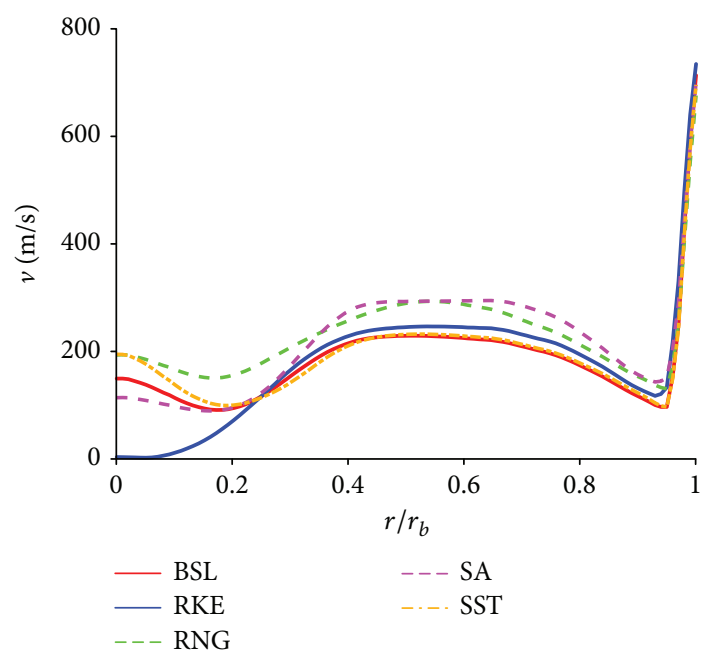

(a) The magnitude of velocity

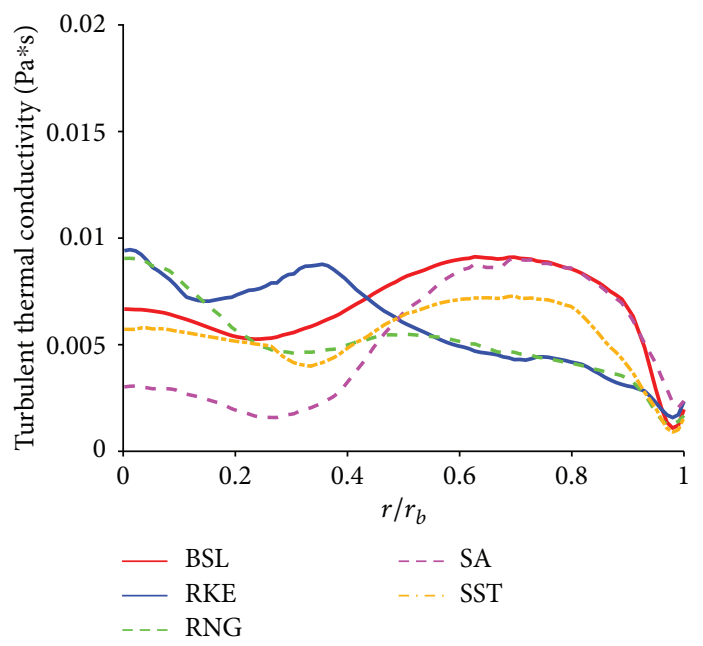

(b) Turbulent thermal conductivity

Figure 11: Parameter distributions in the wall jet.

have managed to reflect the central peak, although lower than the experiment by more than $1 / 3$ due to the exclusion of finite-rate chemistry. This is correlated to the mean temperature discrepancies shown in Figure 8, where the reverse flow temperature of frozen flow is generally lower than the reaction flow. Comparing with BSL and RNG, the SST model has provided the highest central heat flux by about $20 \%$ that of the other two models, while the RNG model has exhibited higher estimation in $r / r_{b}>0.4$. For reaction flow, although a moderate increase occurred in heat flux for RKE, the overall distribution was still frustrating. This is attributed to the poorly estimated reverse flow velocity, which reduced the convection level near the base, and to the underpredicted reverse flow temperature, as shown in Figures 8 and 9. Apart from RKE, all the turbulence models have performed well in finite-rate chemistry simulation, as the central peak was indicated qualitatively and good agreement was shown in $r / r_{b}>0.6$, although the numerical heat flux was overestimated at around $r / r_{b}=0.4$. It is unexpected that the SA model has outperformed in reaction flow than in frozen flow, especially in the central area. This is due to the substantial increase of reverse flow temperature, indicating significant afterburning. The underpredicted central peak may be attributed to the underestimated reverse flow velocity as well as the relatively lower turbulent thermal conductivity near the central area. Furthermore, BSL and SST have performed nearly consistently in base flux prediction, both of which have agreed excellently with the experimental data. The BSL model has provided more accurate central peak of heat flux, while the SST model has performed better more outside with a mild overprediction at the center. Moreover, the RNG model still exhibited higher numerical heat flux in $r / r_{b}>0.4$ than others, especially near the bottom edge.

4.3.2. Radial Temperature and Pressure. Figure 14 compares the numerical temperature and pressure distribution with the experimental data at the wind tunnel test sensors. The thermocouples to acquire local gas temperature extended $12.7 \mathrm{~mm}$ above the base plate, and the pressure transducers were inserted on the surface. It has been indicated that nearly all the turbulence models have generally underpredicted the radial temperature, especially in $r / r_{b}>0.4$. The central temperature estimated by RKE was the most moderate as expected. However, it is unexpected that the SA model has relatively better performed in temperature prediction than the others in $r / r_{b}=0 \sim 0.2$, although the BSL and SST 


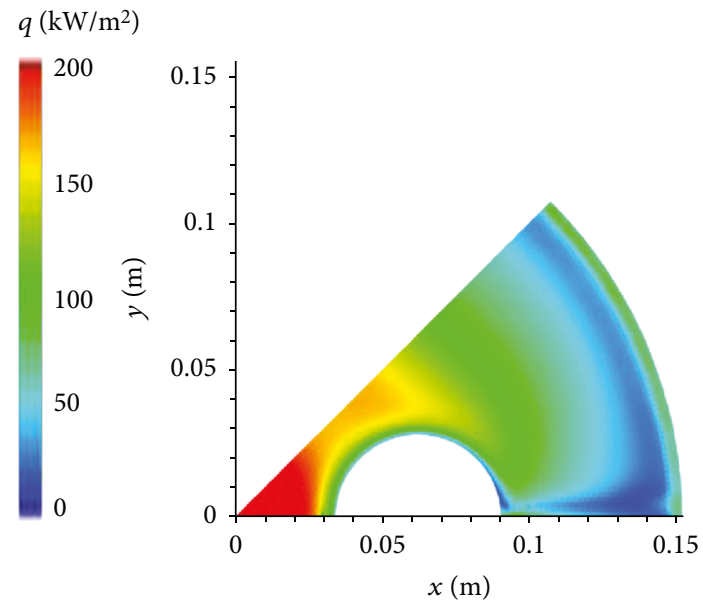

(a) BSL

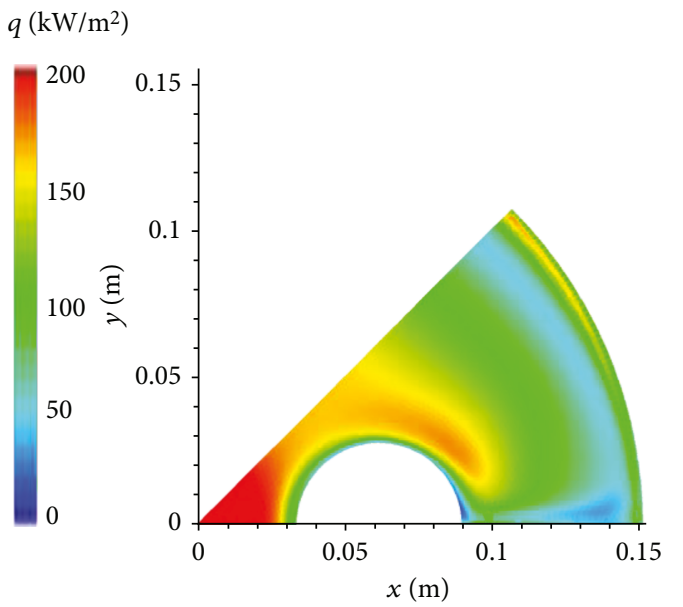

(c) RNG

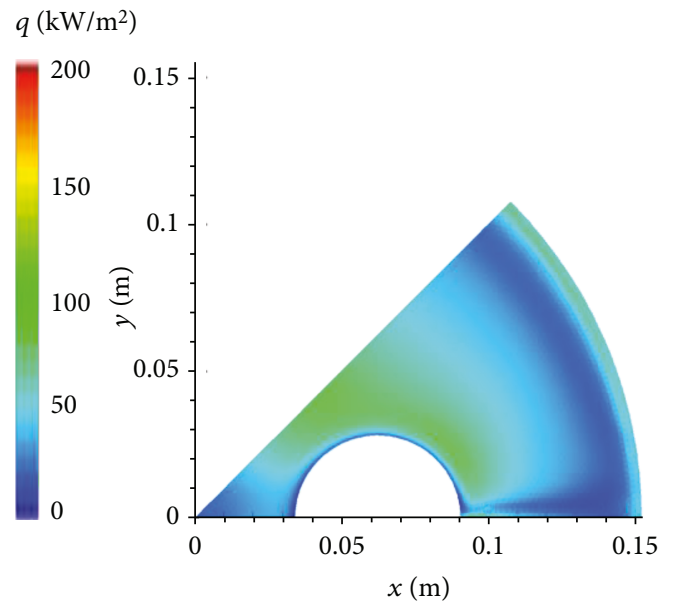

(b) RKE

$q\left(\mathrm{~kW} / \mathrm{m}^{2}\right)$

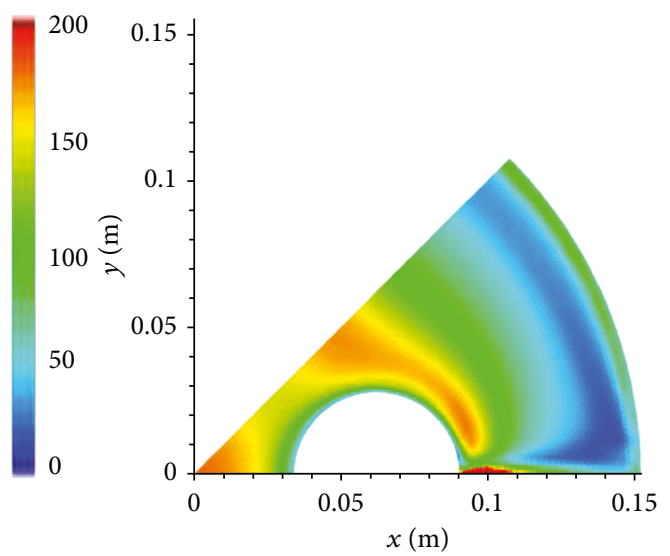

(d) SA

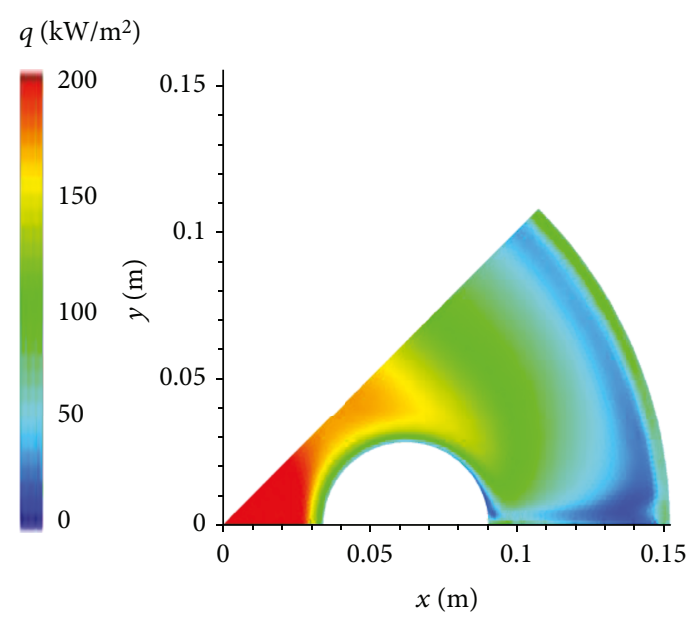

(e) SST

FIGURE 12: Contours of base heat flux for various turbulence models.

models have outperformed it in heat flux prediction. The outperformance of SA exactly corresponds to the higher reverse flow temperature but the lower convective heat transfer as illustrated above. Moreover, the RNG model is the only one that has agreed preferably with the experimen- tal gas temperature in $r / r_{b}>0.6$. The excellent performance of RNG on base temperature estimation may be attributed to its unique formulation of turbulent Prandtl number $\operatorname{Pr}_{t}$ based on the mathematics of renormalization group concept, which correlates $\operatorname{Pr}_{t}$ with laminar Prandtl number Pr. 


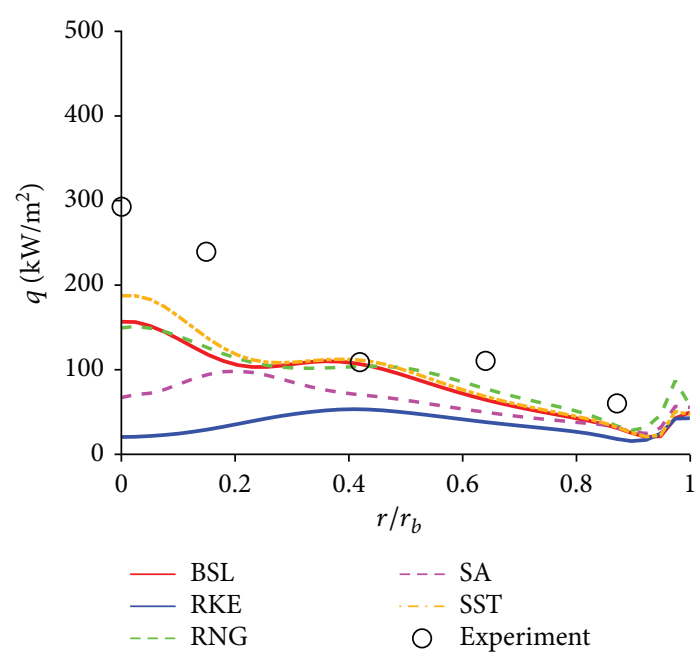

(a) Frozen flow

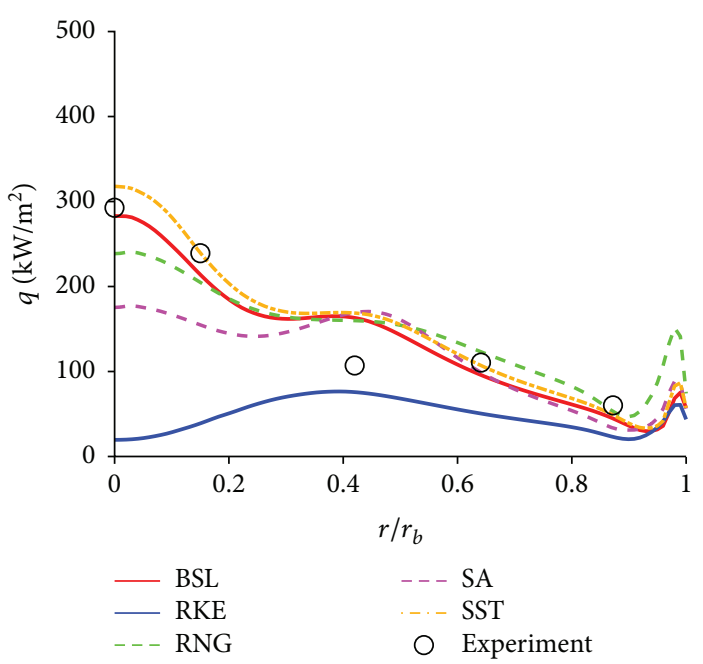

(b) Reaction flow

Figure 13: Radial heat flux distribution on the base.

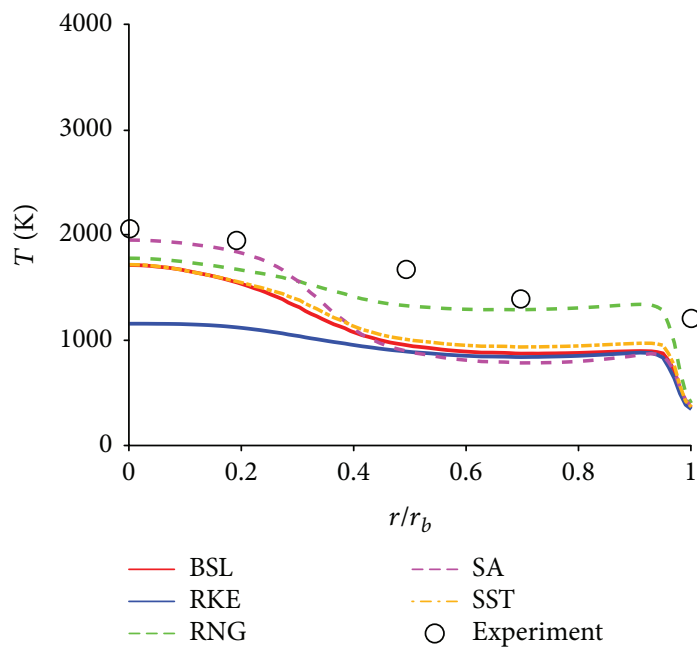

(a) Temperature

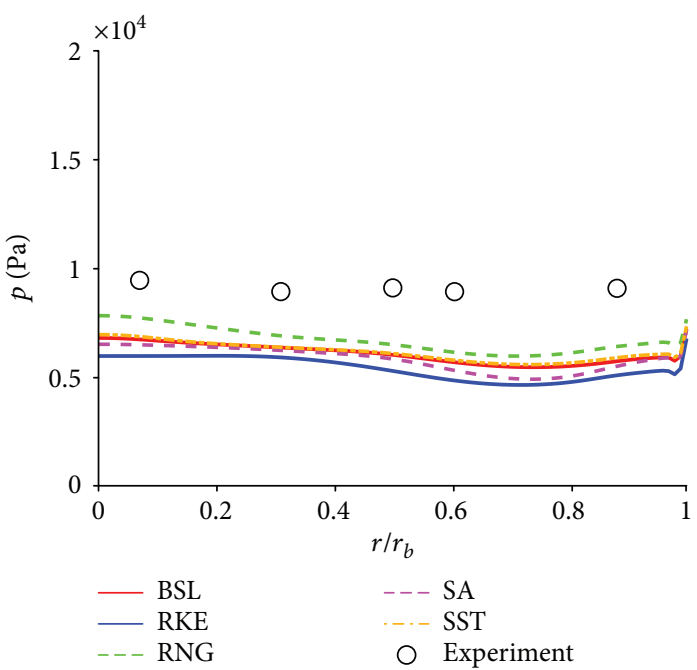

(b) Pressure

FIGURE 14: A comparison of radial temperature and pressure at sensors.

Besides, the BSL and SST models have only indicated the qualitative radial distribution of temperature and have underestimated the central peak by more than $15 \%$. Given the favorable agreement in radial base heat flux, the underestimation in temperature implies the overprediction on convective gas-to-base heat transfer.

Radial pressure on the base plate is shown in Figure 14(b). All the turbulence models have underpredicted it by more than $15 \%$, which was considered due to the overestimation of freestream entrainment into the base region as well as the discrepancies in the ambient pressure surrounding the base [13]. Relatively, the RNG model has performed best in pressure prediction while the RKE model has poorly estimated it, which is more than $20 \%$ lower than that of RNG, and the other three models have just performed well qualitatively.

\section{Conclusion}

The base heating of a four-nozzle rocket has been simulated using a density-based steady state solver to solve the Reynolds-averaged Navier-Stokes (RANS) equations. Five different RANS turbulence models have been used for comparison with finite-rate chemistry involved to simulate afterburning. Parameter consistency at the nozzle exit has been suggested among the turbulence models, while each turbulence model has provided distinct turbulent viscosity distributions. The base flow structures, including plume collision, reverse flow, impingement, wall jet, and the derived recirculation vortices, have been shown by all the turbulence models except RKE.

The plume collision pressure and reverse flow velocity have been compared. The BSL and SST models have estimated 
the highest collision pressure while the RNG model has provided the farthest collision point from the bottom. Nevertheless, the reverse point was nearly identical to that of BSL and SST due to the relatively lower collision pressure of RNG. The RKE model has underpredicted the reverse flow velocity due to the excessive turbulence dissipation at the large-stain area. Furthermore, the increase of reactive base flow temperature and base heat flux has indicated the heat release of chemical reactions compared to the frozen flow results. The SA model has provided the highest reverse flow temperature while the RKE model has significantly underestimated it. In the recirculation region, RNG has estimated the highest temperature for more involvement of plume. For wall jet temperature, the SA model has performed better near-base center while the RNG model has best predicted the overall distribution. All the turbulence models of interest have underestimated base pressure due to overpredictions on air entrainment and discrepancies in freestream pressure conditions. Finally, the BSL and SST models have favorably approximated base heat flux compared to experimental data, respectively, having performed better in the central peak and at the larger radius. In conclusion, Menter's BSL and SST models are appropriate to predict launch vehicle base heating, but the RKE model is unsuitable. The present work might help the selection of the turbulence model in computations on base heating.

\section{Nomenclature}

L: Axial distance from the base to the nozzle exit plane

$D_{e}$ : Diameter of the nozzle exit

$D_{s}$ : Nozzle spacing

$D_{t}$ : Diameter of nozzle throat

$r_{b}$ : Radius of rocket bottom plate

$\rho$ : Density

A: Preexponential factor of Arrhenius equation

$b$ : Exponent of temperature

Ea: Activation energy

$\mu$ : Dynamic viscosity

$v$ : Kinetic viscosity

$k$ : Turbulence kinetic energy

$\varepsilon$ : Turbulent dissipation rate

$\omega$ : Specific dissipation rate

q: Wall heat flux

$r_{e}$ : Radius of the nozzle exit

Pr: Laminar Prandtl number

$\mathrm{Pr}_{t}$ : Turbulent Prandtl number

$\kappa_{t}$ : Turbulent thermal conductivity.

\section{Subscripts}

b: Base

e: Nozzle exit

$s$ : Nozzle spacing

$t$ : Nozzle throat or turbulence.

\section{Data Availability}

The experimental data used to support the findings of this study are included within the Ref. [4].

\section{Disclosure}

The present work originates from the research on launch vehicle base heating in a project of China Academy of Launch Vehicle Technology.

\section{Conflicts of Interest}

The authors declare that they have no conflicts of interest.

\section{Acknowledgments}

Thanks are due to the support and instructions from its researchers and from my tutor Dr. Yi Jiang.

\section{References}

[1] C. R. Mullen, R. L. Bender, R. L. Bevill, J. Reardon, and L. Hartley, Saturn Base Heating Handbook, NASA, 1972, CR-61390.

[2] B. H. Goethert and L. T. Barnes, Some Studies of the Flow Pattern at the Base of Missiles with Rocket Exhaust Jets, , Defense Technical Information Center, , 1980, AEDC-TR58-12.

[3] T. F. Greenwood, Y. C. Lee, R. L. Bender, and R. E. Carter, "Space shuttle base heating," Journal of Spacecraft and Rockets, vol. 21, no. 4, pp. 339-345, 1984.

[4] N. T. Musial and J. J. Ward, Base Flow Characteristics for Several Four Clustered Rocket Configurations at Mach Numbers from 2.0 to 3.5, NASA, 1961, TN-D-1093.

[5] M. Sillen, "Investigation of base flow on a space rocket with plumes," in 8th AIAA International Space Planes and Hypersonic Systems and Technologies Conference, Norfolk, VA, USA, April 1998.

[6] B. E. Launder and B. I. Sharma, "Application of the energydissipation model of turbulence to the calculation of flow near a spinning disc," Letters in Heat and Mass Transfer, vol. 1, no. 2, pp. 131-137, 1974.

[7] R. Webster, "Numerical study of base flow induced by exhaust plume impingement," in 34th AIAA/ASME/SAE/ASEE Joint Propulsion Conference and Exhibit, Cleveland, OH, USA, July 1998.

[8] B. S. Baldwin and H. Lomax, "Thin-layer approximation and algebraic model for separated turbulent flows," in 16th Aerospace Sciences Meeting, pp. 78-257, Huntsville, AL, U.S.A., January 1978.

[9] S. Zhang, J. Liu, and X. Zhao, "An integrated CFD tool for lunch vehicles base-heating analyses," in 42nd AIAA/ASME/SAE/ASEE Joint Propulsion Conference \& Exhibit, Sacramento, California, July 2006.

[10] R. Nallasamy, M. Kandula, L. Duncil, and P. Schallhorn, "Numerical simulation of the base flow and heat transfer characteristics of a four-nozzle clustered rocket engine," in 40th Thermophysics Conference, Seattle, Washington, USA, June 2008.

[11] B. S. Balwin and T. J. Barth, "One-equation turbulence transport model for high Reynolds number wall bounded flows," in NASA TM-102847, Reno, NV, USA, 1990.

[12] H. Negishi, N. Yamanishi, M. Arita, E. Namura, and S. Ohkubo, "Numerical analysis of plume heating environment for H-IIA launch vehicle during powered ascent," in 
43rd AIAA/ASME/SAE/ASEE Joint Propulsion Conference \& Exhibit, Cincinnati, OH, USA, July 2007.

[13] M. Mehta, F. Canabal, S. B. Tashakkor, and S. D. Smith, "Numerical Base heating sensitivity study for a four-rocket engine core configuration," Journal of Spacecraft and Rockets, vol. 50, no. 3, pp. 509-526, 2013.

[14] D. Patel, "Antares liquid rocket engine convective base heating: AJ-26 to RD-181," in AIAA Modeling and Simulation Technologies Conference, Washington, D.C., USA, June 2016.

[15] P. L. Roe, "Characteristic-based schemes for the Euler equations," Annual Review of Fluid Mechanics, vol. 18, no. 1, pp. 337-365, 1986.

[16] T. S. Wang, "Thermophysics characterization of kerosene combustion," Journal of Thermophysics and Heat Transfer, vol. 15, no. 2, pp. 140-147, 2001.

[17] D. Watts, "Assessing computational fluid dynamics turbulence models for rocket exhaust plume simulation," in 52nd AIAA/SAE/ASEE Joint Propulsion, Salt Lake City, UT, USA, July 2016.

[18] P. Spalart and S. Allmaras, "A one-equation turbulence model for aerodynamic flows," in 30th Aerospace Sciences Meeting and Exhibit, Reno, NV, U.S.A., January 1992.

[19] T. H. Shih, W. W. Liou, A. Shabbir, Z. Yang, and J. Zhu, "A new $k$ - $\epsilon$ Eddy viscosity model for high Reynolds number turbulent flows," Computers \& Fluids, vol. 24, no. 3, pp. 227-238, 1995.

[20] S. A. Orszag, V. Yakhot, W. S. Flannery et al., "Renormalization group modeling and turbulence simulations," in International Conference on Near-Wall Turbulent Flows, Tempe, Arizona, USA, 1993.

[21] F. R. Menter, "Two-equation eddy-viscosity turbulence models for engineering applications," AIAA Journal, vol. 32, no. 8, pp. 1598-1605, 1994.

[22] F. R. Menter, M. Kuntz, and R. Langtry, "Ten years of industrial experience with the SST turbulence model," in Turbulence, Heat and Mass Transfer, K. Hanjalic, Y. Nagano, and M. Tummers, Eds., pp. 625-632, Begell House, Inc., 2003.

[23] R. Dutta, A. Dewan, and B. Srinivasan, "Comparison of various integration to wall (ITW) RANS models for predicting turbulent slot jet impingement heat transfer," International Journal of Heat and Mass Transfer, vol. 65, no. 5, pp. 750764, 2013.

[24] S. Chander and A. Ray, "Flame impingement heat transfer: a review," Energy Conversion and Management, vol. 46, no. 1819, pp. 2803-2837, 2005. 


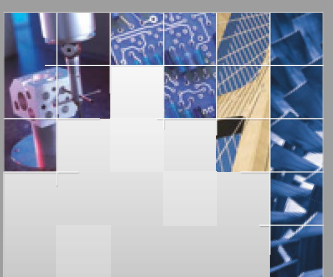

\section{Enfincering}
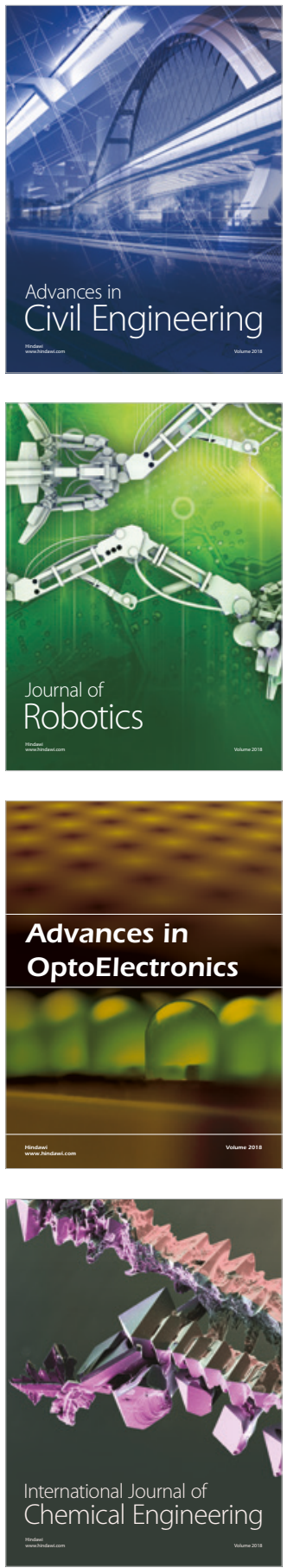

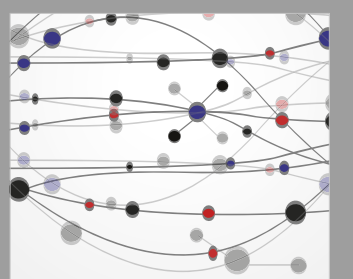

\section{Rotating \\ Machinery}

The Scientific World Journal

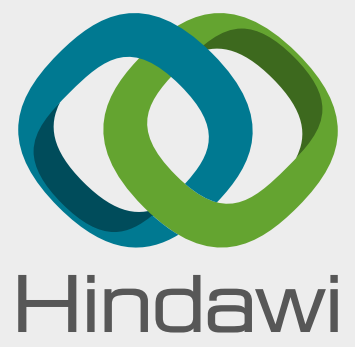

Submit your manuscripts at

www.hindawi.com
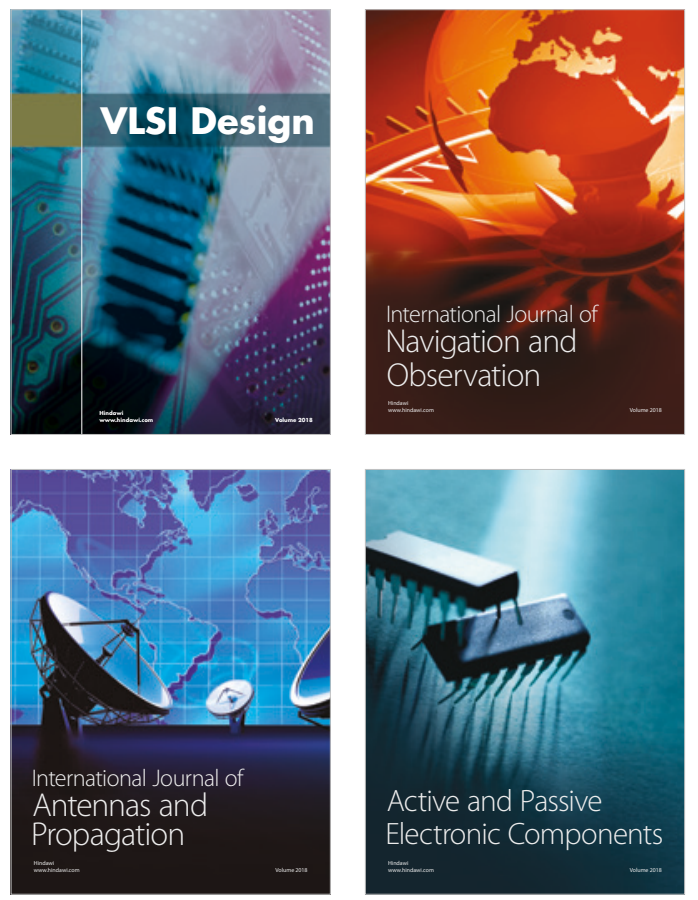
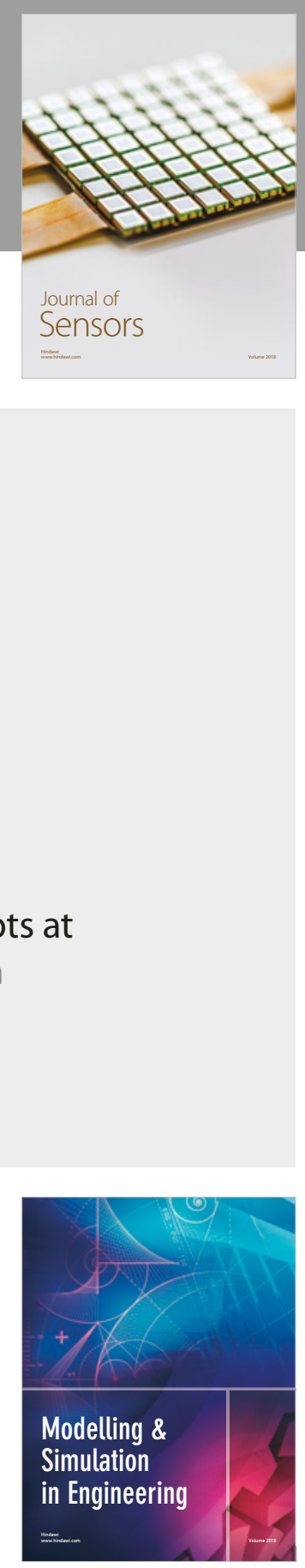

\section{Advances \\ Multimedia}
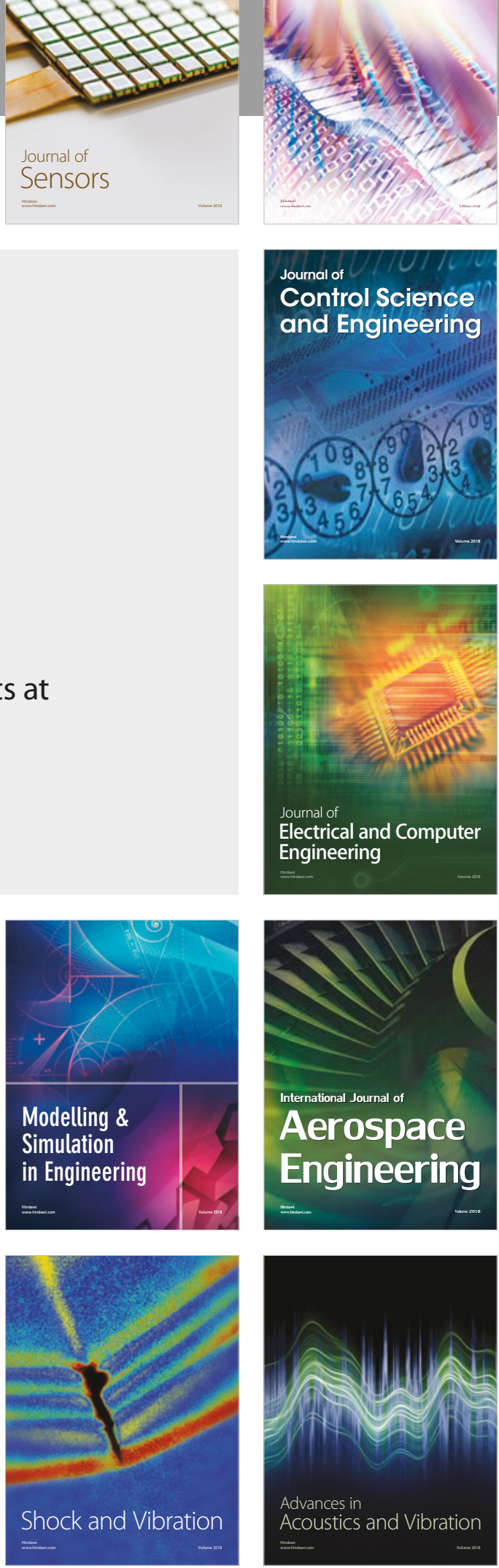